\section{Prevalence and factors associ- ated with memory disturbance and dementia after acute ischemic stroke}

\author{
Jesada Surawan, 1 Teabpaluck \\ Sirithanawutichai, ${ }^{2}$ Suchat Areemit, ${ }^{3}$ \\ Somsak Tiamkao,,,5 Suprawita Saensak ${ }^{2}$
}

1Health Science Program, Faculty of

Medicine, Mahasarakham University,

Mahasarakham, Thailand; 2Department

of Medicine, Faculty of Medicine,

Mahasarakham University,

Mahasarakham, Thailand; ${ }^{3}$ Department

of Surgery, Faculty of Medicine, Khon

Kaen University, Khon Kaen, Thailand;

4North-eastern Stroke Research Group,

Khon Kaen University, Khon Kaen,

Thailand; 5Department of Medicine,

Faculty of Medicine, Khon Kaen

University, Khon Kaen, Thailand

\begin{abstract}
Prevalence and risk factors associated with memory disturbance and dementia were determined in acute ischemic stroke (AIS) patients in hospitals before discharge, three and six months after stroke. A prospective cohort study was conducted during January-December 2017 with 401 AIS patients admitted to Srinagarind Hospital, Khon Kaen Hospital and Chum Phae Hospital, Khon Kaen, Thailand. The demographics and clinical characteristics, previous illness and past medical history, and laboratory test results of the patients were collected from the medical records, while depression screening, NIH stroke scale (NIHSS) scoring and mini mental state examination (MMSE) were performed using particular medical record forms. The prevalence of memory disturbance and dementia was $56.6,41.6$ and $38.2 \%$ before discharge, three and six months after stroke, respectively. Based on logistic regression analysis, age, education and stroke severity were the risk factors associated with the studied disorders before discharge and three months after stroke. Meanwhile, age and education were the risk factors for six months after stroke. Our findings suggested that the prevalence of memory disturbance and dementia remained high at all study periods.
\end{abstract}

\section{Introduction}

Post-stroke dementia (PSD) is any dementia present a few months after stroke, irrespective of its cause and causal relationship with stroke. Stroke is the most common cause of dementia accounting for $66 \%$ of all cases, followed by Alzheimer's disease $(33 \%)$, and a combination of these two disorders and other risk factors $(1 \%)$, such as aging, low education level, diabetes mellitus, atrial fibrillation, myocardial infarction, hypertension, medial temporal lope atrophy and white matter changes. ${ }^{1}$ The prevalence of post-stroke cognitive impairment and dementia ranges from 20 to $80 \% .2,3$ depending on the population studied, the criteria used for the diagnosis of dementia or memory disturbance and the time interval between the stroke and the neuropsychological assessment. ${ }^{3}$ PSD has a profound adverse effect on stroke patients, causing the patients to live with reduced ability or even with disability in their daily life. ${ }^{4}$ The survival rate of stroke patients with dementia has been reported to be 5.1 years while those without dementia show the survival rate of 8.8 years. ${ }^{5}$ Moreover, patients with dementia are likely to have twice the risk of recurrent stroke when compared to those without dementia. ${ }^{6}$ Additionally, dementia is one of the most important factors of mortality in stroke patients, in which the mortality rate of stroke patients with dementia is three times greater than those without dementia.?

In general, the determinants of memory disturbance and dementia in stroke patients include: (i) patient demographics and clinical characteristics, such as increasing age, 5 low educational levels $6,8,9$ and cognitive impairment before stroke; ${ }^{6}$ (ii) vascular risk factors, such as diabetes mellitus $6,8,10,11$ hypertension, ${ }^{12}$ atrial fibrillation, ${ }^{12}$ myocardial infarction, ${ }^{1}$ smoking $5,6,11$ and alcohol consumption; 13 (iii) stroke factors, such as previous stroke, ${ }^{8}$ recurrent stroke; 8 (iv) stroke complication, including incontinence, acute confusion, hypoxic ischemic episodes and early seizures; ${ }^{8}$ and (v) abnormalities on brain imaging, such as leukoaraiosis, atrophy, medial temporal lope atrophy, size and number of lesions and white matter changes. 8,10 The main mechanisms of post-stroke dementia are the direct effect of stroke, such as lesions on small cerebral vessels which result in cerebral micro bleeds (CMBs) and may be related to delayed memory impairment. Neuroanatomical lesions, such as hippocampal sclerosis and white matter lesions (WMLs), may also contribute to dementia and post-stroke dementia associated with
Correspondence: Somsak Tiamkao, Department of Medicine, Faculty of Medicine, Khon Kaen University, Khon Kaen 40002, Thailand.

Tel.: +66.81.050.4626 - Fax: +66.43.363.664 E-mail: somtia@kku.ac.th

Key words: Acute ischemic stroke; dementia; memory disturbance; prevalence.

Acknowledgments: this work was supported by the Graduate Studies Division, Faculty of Medicine, Mahasarakham University; and the North-eastern Stroke Research Group, Khon Kaen University. The authors would like to thank Prof. K. Sawanyawisuth from the Department of Medicine, Faculty of Medicine, Khon Kaen University for proof-reading the manuscript.

Conflict of interest: the authors declare that they have no conflict of interest.

Received for publication: 27 May 2018.

Revision received: 3 July 2018.

Accepted for publication: 5 July 2018.

This work is licensed under a Creative Commons Attribution NonCommercial 4.0 License (CC BY-NC 4.0).

(C) Copyright J. Surawan et al., 2018

Licensee PAGEPress, Italy

Neurology International 2018; 10:7761

doi:10.4081/ni.2018.7761

asymptomatic Alzheimer pathology. 3

The common symptoms of memory disturbance and dementia, which frequently occur a few months after stroke, include short-term memory impairment, language deficits, mood swings and behavioral changes, or even disability to live daily life. Even though there are a number of treatments currently available for dementia, most of which are developed for palliative purposes and are limited in number. Such treatments include cholinesterase inhibitors used to relieve the symptoms by improving awareness, ${ }^{14,15}$ and brain rehabilitation and constant brain training like memory training, calculations, speech, writing and learning new things. ${ }^{16}$ For this reason, most approaches to memory disturbance and dementia treatments focus on preventing recurrent stroke, controlling and reducing the risk factors for stroke, as well as preventing complications in stroke patients, ${ }^{17}$ which is important.

Many previous studies have reported differences in the prevalence and incidence of cognitive impairment and dementia after stroke. Besides, the causes and risk factors for cognitive impairment and dementia are still debated. Therefore, the purpose of this 
prospective observational cohort study was to determine the prevalence and factors associated with memory disturbance and dementia in acute ischemic stroke (AIS) patients in hospitals before discharge, and after 3 and 6 months of follow-up. This study should provide a foundation for future health service management to reduce the prevalence and risk factors associated with memory disturbance and dementia in AIS patients.

\section{Materials and Methods}

\section{Subject/patient selection}

A prospective observational cohort study was employed in this study. A total of 401 (male: 216 and female: 185) AIS patients admitted to the Stroke Unit of Srinagarind Hospital, Khon Kaen Hospital and Chum Phae Hospital in Khon Kaen province of Thailand from January 1 to December 31, 2017 with a documented diagnosis of AIS were selected.

Stroke patients meeting any of the following criteria were excluded from the study: (i) having a history of depression diagnosis, being at risk of depression as evaluated by $2 \mathrm{Q} / 9 \mathrm{Q}$ depression screening questionnaires or undergoing medications for depression, (ii) having a history of diagnosis or comorbidities or undergoing treatments for vitamin B12 deficiency, renal failure, thyroid hormone deficiency, syphilis, HIV, Pick's disease, CreutzfeldtJakob disease, Huntington's disease, Parkinson's disease, Alzheimer's disease, (iii) having hearing loss, and (iv) having visual or communication impairments. Stroke patients being possibly at risk of worsened symptoms if they participated in the study as suggested by their physicians were also excluded.

\section{Data collection}

A retrospective chart review was then conducted to abstract the following information from each patient included in the study: patient demographics and clinical characteristics (age, sex, education, alcohol consumption, smoking, type of stroke, stroke location, stroke severity, depressive disorder, and memory disturbance and dementia); previous illness and past medical history and comorbidities (atrial fibrillation, prior stroke, myocardial infarction, hypertension, diabetes mellitus, transient ischemic attack, heart failure, hypercholesterolemia, a family history of memory disturbance and dementia, use of drugs that may lead to dementia [antiplatelet agents, anticoagulants, $\beta$-adrenoceptor blocking drugs, positive inotropic drugs, diuretics, vasodilators, centrally acting antihypertensive drugs, $\alpha$-adrenoceptor blocking drugs, angiotensin-converting enzyme inhibitors, angiotensin-II receptor antagonists, nitrates, sodium channel blockers, calcium channel blockers, lipid-regulating drugs, and antidiabetic drugs], and length of hospital stay); and laboratory test results (systolic and diastolic blood pressure, blood sugar level, glycated hemoglobin $\left(\mathrm{HbA}_{1} \mathrm{C}\right)$ level, LDL, HDL and total cholesterol level, triglyceride, and atrial fibrillation and/or other arrhythmia in the EKG). The information was collected from patients in hospitals before discharge, and after 3 and 6 months of follow-up.

\section{Measurements}

\section{Prior stroke, stroke, ischemic brain} infarction, and transient ischemic attack

Prior stroke was diagnosed according to the previous diagnoses or treatments, while stroke was diagnosed according to the World Health Organization (WHO) criteria and according to the International Classification of Diseases and Related Health Problem 10th Revision (ICD10) criteria. ${ }^{18}$ According to $\mathrm{WHO}$, stroke cases must meet the following qualifying criteria: (i) having clinical syndromes which cause a partial or complete loss of cerebral functions, thus leading to death, (ii) having symptoms, caused by stroke, that occur suddenly or last for $>24 \mathrm{~h}$, and (iii) having signs and symptoms indicated by brain imaging techniques (magnetic resonance imaging (MRI) and/or computed tomography (CT) scan). On the other hand, based on the ICD10, stroke cases must meet the following qualifying criteria: (i) having rapidly developing signs of focal (or global) disturbance of cerebral function, leading to death or lasting for $>24 \mathrm{~h}$, with no apparent cause other than vascular causes, ${ }^{19}$ and (ii) having signs and symptoms indicated by CT scan and/or MRI. Stroke severity was assessed using the NIH stroke scale (NIHSS), which consists of 11 questions in which the highest score indicates poor poststroke clinical manifestations and lower scores indicate better post-stroke clinical presentations as follows: $\leq 4=$ mild impairment; $5-14=$ mild to moderate; $15-24=$ severe; and $\geq 25=$ very severe. ${ }^{20}$ Ischemic brain infarction was diagnosed according to the ICD10 criteria, when MRI or CT imaging shows no hemorrhage, which was classified as cardio embolic (CE) if a cardiac source of embolus was found with all other ischemic infarcts classified as atherothrombotic brain infarcts and infarcts of unknown origin.
Transient ischemic attack (TIA) was diagnosed according to the ICD10 criteria, and was as signs and symptoms of neurological disorders caused by stroke, which disappear within $24 \mathrm{~h}$ after the onset of stroke.

\section{Depressive disorder, and memory distur- bance and dementia}

Depressive disorder was evaluated using both two questions $(0=$ normal; $1-2=$ abnormal $)$ and nine questions $(<7=$ normal; $7-12=$ mild; $13-18=$ moderate; and $\geq 19=$ severe $).^{21}$ Memory disturbance and dementia were assessed using the mini mental state examination or MMSE (MMSE Thai-2002), which comprises 11 questions with the highest score of $30 .{ }^{22}$ Patients with the MMSE scores of $\leq$ 23 were classified as having memory disturbance and dementia. Dementia was also diagnosed according to the modified diagnostic and statistical manual of mental disorders $\mathrm{V}$ (DSM-V) criteria. $^{23}$

\section{Hypertension}

Hypertension was diagnosed according to the JNC7 guideline ${ }^{24}$ or according to the previous diagnoses or treatments. Hypertension cases must meet the following qualifying criteria: (i) systolic blood pressure $\geq 140 \mathrm{mmHg}$, and (ii) diastolic blood pressure $\geq 90 \mathrm{mmHg}$. Measurements were performed at least twice after a 10 -min rest.

\section{Diabetes mellitus}

Diabetes mellitus was diagnosed according to the World Health Organization (WHO) criteria ${ }^{25}$ or according to the previous diagnoses or treatments. Diabetes mellitus cases must meet all the following qualifying criteria: (i) fasting plasma glucose (FPG) $\geq 126$ $\mathrm{mg} / \mathrm{dL}$, (ii) casual plasma glucose $\geq 200$ $\mathrm{mg} / \mathrm{dL}$ with symptoms of diabetes, and (iii) plasma glucose $\geq 200 \mathrm{mg} / \mathrm{dL}$ after $2 \mathrm{~h}$ of oral glucose tolerance test (OGTT). All measurements were performed at least twice.

\section{Hypercholesterolemia}

Hypercholesterolemia was diagnosed according to the National Cholesterol Education Program (NCEP) and Adult Treatment Panel III (ATPIII) criteria. ${ }^{26}$ Hypercholesterolemia cases must meet all the following qualifying criteria: (i) total cholesterol level $>240 \mathrm{mg} / \mathrm{dL}$, (ii) LDL cholesterol level $>160 \mathrm{mg} / \mathrm{dL}$, (iii) HDL cholesterol level $<40 \mathrm{mg} / \mathrm{dL}$, and (iv) triglyceride $>200$ $\mathrm{mg} / \mathrm{dL}$.

\section{Statistical analyses}

The demographics and frequencies of patients are expressed as the number and percentage. Comparisons of dichotomous and categorical variables were conducted using Chi-square $\left(\chi^{2}\right)$ analysis with Fisher's exact test used for comparisons with the expected cells of $<5$ exceeding $20 \%$. Comparisons of 
continuous data were performed using t-tests for normally distributed variables and Mann Whitney $U$ tests on variables that did not meet the assumption of normality. Multivariate analysis and backward stepwise logistic regressions were carried out to identify the independent variables associated with memory disturbance and dementia after AIS. Several exposure variables were included in multiple conditional logistic regression analysis, and the results are expressed as the approximate relative risk (odds ratio; OR) and $95 \%$ confidence interval. The $P$ values of $<0.05$ were considered to be statistically significant in all analytical procedures. All analyses were performed using STATA v 10.0 (StataCorp, College Station, TX, USA).

\section{Results}

\section{Patient demographics, clinical char- acteristics and stroke prevalence}

The patient demographics, previous illness and past medical history, and laboratory test results and clinical characteristics of AIS patients with and without memory disturbance and dementia are given in Tables 1-3. As shown in Table 1, it was found that the number of male AIS patients (216, 53.9\%) was greater than that of female AIS patients $(185,46.1 \%)$, and the prevalence of memory disturbance and dementia was highest $(56.6 \%)$ in AIS patients in hospitals before discharge, followed by those undergoing fellow-up evaluations after three (41.6\%) and six $(38.2 \%)$ months of discharge. Based on the patient demographics, significant differences $(P>0.05)$ were not observed between AIS patients with and without memory disturbance and dementia with regard to sex, alcohol consumption, smoking and stroke location. By contrast, these two patient groups showed significant differences $(P<0.05)$ in age, education and type of stroke (Table 1).

According to the previous illness and past medical history, there were no significant differences $(P>0.05)$ between stroke patients with and without memory disturbance and dementia with respect to hospital length of stay, prior stroke, diabetes mellitus, transient ischemic attack, hypercholesterolemia and use of drugs. Meanwhile, significant differences $(P<0.05)$ were observed for atrial fibrillation and hypertension (Table 2).

Table 1. Prevalence of memory disturbance and dementia in AIS patients based on the selected patient demographics $(\mathrm{n}=401)$. The results are expressed as the number with the percentage in brackets or as the median, min-max.

\begin{tabular}{|c|c|c|c|}
\hline Patient demographics & $\begin{array}{l}\text { Dementia } \\
(\mathrm{n}=227)\end{array}$ & $\begin{array}{l}\text { Non-dementia } \\
\quad(n=174)\end{array}$ & P-value \\
\hline $\begin{array}{l}\text { Sex } \\
\text { Male } \\
\text { Female }\end{array}$ & $\begin{array}{l}115(50.7 \%) \\
112(49.3 \%)\end{array}$ & $\begin{array}{l}101(58.1 \%) \\
73(41.9 \%)\end{array}$ & 0.141 \\
\hline Age (years) & $69,20-92$ & $58,23-83$ & $<0.001^{*}$ \\
\hline $\begin{array}{l}\text { Education levels } \\
\text { Uneducated/primary } \\
\text { Secondary and higher }\end{array}$ & $\begin{array}{c}193(85.0) \\
34(15.0 \%)\end{array}$ & $\begin{array}{l}93(53.5 \%) \\
81(46.5 \%)\end{array}$ & $<0.001^{*}$ \\
\hline Alcohol consumption & $84(37.0 \%)$ & $71(40.8 \%)$ & 0.439 \\
\hline Smoking & $73(32.2 \%)$ & $66(37.9 \%)$ & 0.229 \\
\hline $\begin{array}{l}\text { Types of stroke }(n=400) \\
\text { Thrombosis } \\
\text { Embolism }\end{array}$ & $\begin{array}{c}183(81.0 \%) \\
43(19.0 \%)\end{array}$ & $\begin{array}{c}156(89.7 \%) \\
18(10.3 \%)\end{array}$ & $0.017^{*}$ \\
\hline $\begin{array}{l}\text { Stroke location }(\mathrm{n}=346) \\
\text { Left } \\
\text { Right } \\
\text { Both sides }\end{array}$ & $\begin{array}{c}86(44.1 \%) \\
98(50.3 \%) \\
11(5.6 \%) \\
\end{array}$ & $\begin{array}{c}82(54.3 \%) \\
60(39.7 \%) \\
9(6.0 \%) \\
\end{array}$ & 0.142 \\
\hline
\end{tabular}

Table 2. Prevalence of memory disturbance and dementia in AIS patients based on the previous illness and past medical history ( $\mathrm{n}=$ 401). The results are expressed as the number with the percentage in brackets or as the median, min-max.

\begin{tabular}{|c|c|c|c|}
\hline Previous illness/past medical history & $\begin{array}{l}\text { Dementia } \\
(\mathrm{n}=227)\end{array}$ & $\begin{array}{l}\text { Non-dementia } \\
\quad(\mathrm{n}=174)\end{array}$ & P-value \\
\hline Having a family history of the disorders & $19(8.4 \%)$ & $14(8.1 \%)$ & 0.907 \\
\hline Hospital length of stay & $2,1-16$ & $2,1-9$ & 0.064 \\
\hline Atrial fibrillation & $37(16.3 \%)$ & $15(8.6 \%)$ & $0.023 *$ \\
\hline Prior stroke & $30(13.2 \%)$ & $26(14.9 \%)$ & 0.621 \\
\hline Hypertension & $130(57.3 \%)$ & $77(44.3 \%)$ & $0.010^{*}$ \\
\hline Diabetes mellitus & $72(31.7 \%)$ & $49(28.2 \%)$ & 0.442 \\
\hline Transient ischemic attack & $5(2.2 \%)$ & $9(5.2 \%)$ & 0.108 \\
\hline Hypercholesterolemia & $79(34.8 \%)$ & $57(32.8 \%)$ & 0.668 \\
\hline \multicolumn{4}{|l|}{ Use of drugs } \\
\hline Antiplatelet agents & $55(24.2 \%)$ & $37(21.3 \%)$ & 0.484 \\
\hline Anticoagulants & $30(13.2 \%)$ & $14(8.1 \%)$ & 0.101 \\
\hline$\beta$-adrenoceptor blocking drugs & $14(6.2 \%)$ & $9(5.2 \%)$ & 0.671 \\
\hline Diuretics & $60(26.4 \%)$ & $40(22.9 \%)$ & 0.430 \\
\hline Angiotensin-converting enzyme inhibitors & $20(8.8 \%)$ & $12(6.9 \%)$ & 0.483 \\
\hline Calcium channel blockers & $31(13.7 \%)$ & $14(8.1 \%)$ & 0.078 \\
\hline Lipid-regulating drugs & $79(34.8 \%)$ & $57(32.8 \%)$ & 0.668 \\
\hline Diabetes mellitus & $72(31.7 \%)$ & $48(27.6 \%)$ & 0.371 \\
\hline
\end{tabular}

Asterisks indicate P-values $<0.05$. 
As with the laboratory test results and clinical characteristics, significant differences $(P>0.05)$ in systolic blood pressure, blood sugar level, $\mathrm{HbA}_{1} \mathrm{C}$ level, HDL cholesterol level and triglyceride were not detected between stroke patients with and without memory disturbance and dementia. Similarly, no significant differences $(P<0.05)$ in stroke severity were observed for the two patient groups. By contrast, these two patient groups showed significant differences $(P<0.05)$ in diastolic blood pressure, total cholesterol level, LDL cholesterol level and electrocardiogram, which were consistent with irregular heartbeats, as presented in Table 3.

\section{Risk factors associated with memory disturbance and dementia}

Tables 4 and 5 summarize the major risk factors associated with memory disturbance and dementia in AIS patients at three time intervals (before discharge, and after three and six months of discharge). Evaluations for memory disturbance and dementia were carried out in 401 AIS patients in hospitals before discharge. Only 361 out of 401 patients $(90.0 \%)$ could participate in the evaluations for memory disturbance and dementia after three months of discharge, as 12 patients (3.0\%) died before the scheduled evaluations and 28 patients $(7.0 \%)$ were unable to contact. After six months of discharge, only 361 patients $(90.0 \%)$ could participate in the evaluations. The results showed that the prevalence of memory disturbance and dementia in AIS patients in hospitals before discharge, and after three and six months of discharge was 56.6, 41.6 and $38.2 \%$, respectively.

Logistic regression analysis was conducted to identify independent variables associated with memory disturbance and dementia after AIS, with patients with or without memory disturbance and dementia designated as the dichotomous dependent variables. It was found that independent variables with significant differences in the univariate analysis $(P<0.05)$ were age, education, type of stroke, atrial fibrillation, hypertension, EKG, and stroke severity as observed for AIS patients in hospitals before discharge. At the three-month follow-up evaluation, age, education, hypertension, stroke severity and depression were the major risk factors. Meanwhile, age, education, atrial fibrillation, hypertension and stroke severity were included as the risk factors at the six-month follow-up evaluation (Table 4).

According to the multivariable analysis, it was observed that in AIS patients in hospitals before discharge, the factors associated with memory disturbance and dementia were age, education and stroke severity. While age, education and stroke severity were the factors associated with the studied disorders at the three-month follow-up evaluation, the factors determined at the sixmonth follow-up evaluation included age and education (Table 5).

\section{Discussion}

In the present study, the prevalence of post-stroke memory disturbance and dementia was observed to be highest in AIS patients before discharge $(56.6 \%)$, followed by those undergoing follow-up evaluations after three (41.6\%) and six (38.2\%) months of discharge.

Although this study and many previous ones shared the identical design as prospective hospital-based cohort studies focusing on a population of patients, evaluation of
AIS patients in hospitals before discharge was not included in these studies. 27,28 In addition, MMSE, which is the most popular tool widely used for evaluation of memory disturbance and dementia, ${ }^{27}$ was employed in this study.

The results obtained in this study unraveled that the highest $(56.6 \%)$ prevalence of the studied disorders was observed in AIS patients in hospitals before discharge, which was very common due to the high number AIS patients with the moderate-tohigh $(19.7 \%)$ severity of stroke coming in for examination. Moreover, AIS patients with memory disturbance and dementia were found to develop depressive disorder in spite of a low level of $6.5 \%$, which was well supported by one previous study 29 which elucidated that depression was related to dementia.

In this study, age, education and stroke severity were found to be the major risk factors associated with memory disturbance and dementia in AIS patients before discharge and after three months of follow-up, while age and education were the risk factors after six months of follow-up. The results obtained from this study revealed that AIS patients with increasing age were at greater risk of memory disturbance and dementia, which was consistent with previous studies. $1,8,30$ The findings obtained in this study also showed that AIS patients with low education level were likely to develop the studied disorders, which was in agreement with earlier studies.1,8,30 Moreover, one previous study suggested that high education level was one of the factors associated with lower risk of developing cognitive impairment and dementia [9]. Additionally, the severity of stroke associated with memory disturbance and dementia found in this study was similar to that reported by previous studies. $1,8,30$

Table 3. Laboratory test results and clinical characteristics of AIS patients with and without dementia $(\mathrm{n}=401)$. The results are expressed as the median, min-max or as the number with the percentage in brackets.

\begin{tabular}{|c|c|c|c|}
\hline $\begin{array}{l}\text { Laboratory test results/ } \\
\text { clinical characteristics }\end{array}$ & $\begin{array}{l}\text { Dementia } \\
(\mathrm{n}=227)\end{array}$ & $\begin{array}{l}\text { Non-dementia } \\
(\mathrm{n}=174)\end{array}$ & P-value \\
\hline \multicolumn{4}{|l|}{ Blood pressure (mmHg) } \\
\hline Systolic & $140,96-220$ & $142,95-236$ & 0.259 \\
\hline Diastolic & $79,52-117$ & $84,59-126$ & $0.024 *$ \\
\hline Blood sugar levels $(\mathrm{mg} \% ; n=366)$ & $99,51-392$ & $98,65-341$ & 0.527 \\
\hline HbAlC levels $(\% ; n=255)$ & $6.1,4.4-19.7$ & $6.1,4.3-13.7$ & 0.751 \\
\hline \multicolumn{4}{|l|}{ Cholesterol levels (mg/dl) } \\
\hline Total $(\mathrm{n}=347)$ & $170,71-522$ & $181,85-390$ & $0.007^{*}$ \\
\hline LDL $(n=348)$ & $112,21-244$ & $125,50-317$ & $0.003^{*}$ \\
\hline HDL $(\mathrm{n}=342)$ & $40,18-92$ & $42,18-120$ & 0.112 \\
\hline Triglyceride (mg/dl; $\mathrm{n}=347$ ) & $122,40-613$ & $121,39-726$ & 0.858 \\
\hline EKG (atrial fibrillation) abnormality & $36(70.6 \%)$ & $15(29.4 \%)$ & $0.031^{*}$ \\
\hline NIHSS scores & $3,0-23$ & $1,0-9$ & $<0.001^{*}$ \\
\hline
\end{tabular}

Asterisks indicate P-values $<0.05$. 
This study provided several advantages: (i) a prospective observational cohort study was appropriately used to find out risk factors associated with memory disturbance and dementia, in which the up-to-date data of patients were obtained for the analyses based on time intervals; (ii) acquisition of the data was performed at three time intervals to predict trends in the prevalence of memory disturbance and dementia; and (iii) various risk factors were included for the analyses based on time intervals. However, some restrictions were encountered in this study, including (i) memory disturbance or dementia might develop before AIS in elderly patients aged $\geq 70$ years; and (ii) the tools used might not be appropriate for some cases because of the complexity of the tools. As with the results obtained in this study, patient care should be promoted by relevant agencies in order to reduce risk factors for developing memory disturbance and dementia in stroke patients. Additionally, the knowledge of cerebrovascular diseases, particularly post-stroke memory disturbance and dementia, should be publicly disseminated in order to promote public awareness of the disorders.

\section{Conclusions}

This research has highlighted the prevalence and risk factors that are associated with post-stroke memory disturbance and dementia in AIS patients admitted to three hospitals (Srinagarind Hospital, Khon Kaen Hospital and Chum Phae Hospital) in Khon Kaen province of Thailand during JanuaryDecember 2017. The results showed that the prevalence of memory disturbance and dementia was $56.6 \%, 41.6 \%$ and $38.2 \%$ as observed in AIS patients in hospitals before discharge, and after 3 and 6 months of follow-up, respectively. It was also found that age, education and stroke severity were the major risk factors associated with the studied disorders. Future studies should be directed towards the clearer identification of risk factors to develop risk scores for the prognostication of memory disturbance and dementia after AIS in each time period.

\section{Ethical standards}

This study was approved by the Ethics Committee on Human Research at Mahasarakham University (05/2560), Khon Kaen University (HE601146), Khon Kaen Hospital (KE60024) and Khon Kaen Provincial Health Office (KEC594) based on the ethical standards and procedures. Patients and relatives were informed of inclusion in the registries at the time of
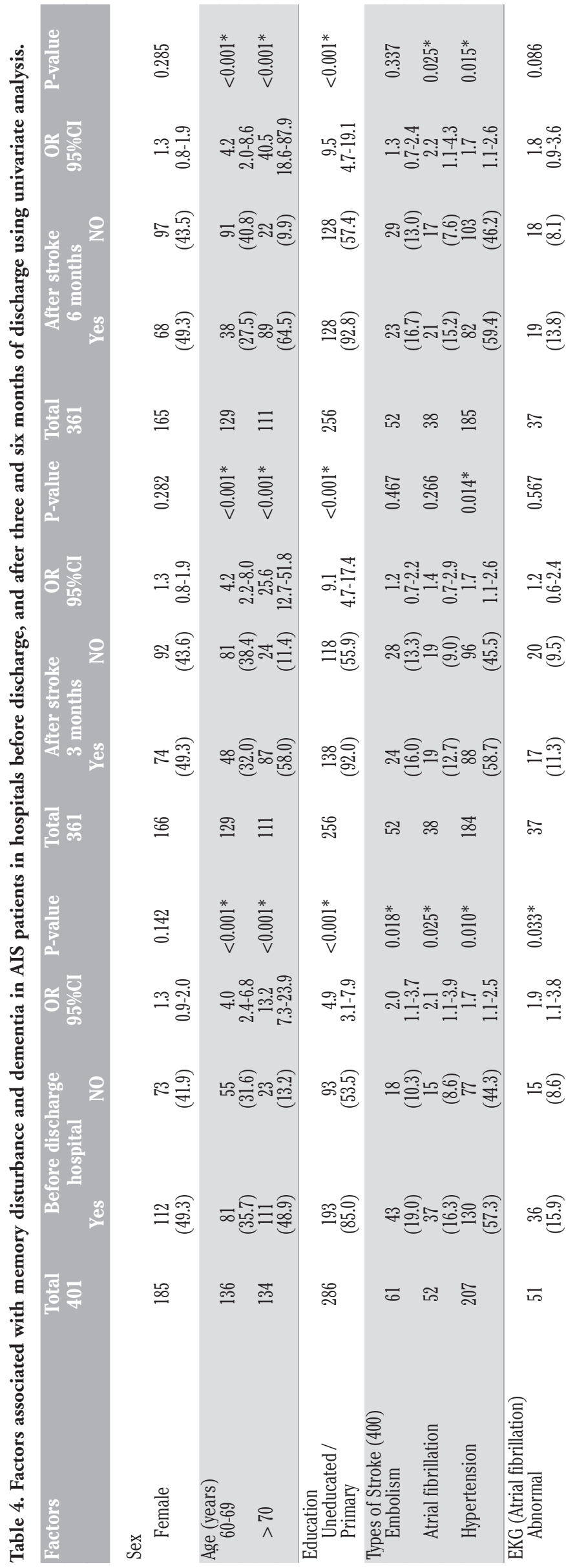
diagnosis and could decline participation or withdraw consent.

\section{References}

1. Leys D. Poststroke dementia. Lancet Neurol 2005;4:752-9.

2. Mellon L. Cognitive impairment six months after ischaemic stroke: a profile from the ASPIRE-S study. BMC Neurol 2015;15:31.

3. Sun J-H, Tan L, Yu J-T. Post-stroke cognitive impairment: epidemiology, mechanisms and management. Ann Transl Med 2014;2:8

4. Xu Y, Yang J, Shang H. Meta-analysis of risk factors for Parkinson's disease dementia. Transl Neurodegener 2016;5:11

5. Melkas S. Poststroke dementia predicts poor survival in long-term follow-up: influence of prestroke cognitive decline and previous stroke. J Neurol Neurosurg Psychiat 2009;80:865-70.

6. Sibolt G. Poststroke dementia is associated with recurrent ischaemic stroke. $\mathrm{J}$ Neurol Neurosurg Psychiatr 2013;2012:304084.

7. Tatemichi T. Dementia after stroke is a predictor of long-term survival. Stroke 1994;25:1915-9.

8. Pendlebury ST, Rothwell PM. Prevalence, incidence, and factors associated with pre-stroke and post-stroke dementia: a systematic review and meta-analysis. Lancet Neurol 2009;8:1006-18.

9. Mirza SS. Higher education is associated with a lower risk of dementia after a stroke or TIA. The Rotterdam Study. Neuroepidemiol 2016;46:120-7.

10. Yang J. Risk factors for incident dementia after stroke and transient ischemic attack. Alzheim Dement 2015;11:16-23.

11. Al-Qazzaz NK. Cognitive assessments for the early diagnosis of dementia after stroke. Neuropsychiatr Dis Treat 2014;10:1743.

12. Béjot Y. Prevalence of early dementia after first-ever stroke a 24-year population-based study. Stroke 2011;42:60712.

13. Choi S-J. Prevalence of Alzheimer's dementia and its risk factors in community-dwelling elderly Koreans. Psychiat Invest 2008;5:78-85.

14. Wilkinson D. Donepezil 308 Study Group: Donepezil in vascular dementia. Neurology 2003;61:479-86.

15. Kurz A. Long $\square$ term safety and cognitive effects of galantamine in the treatment of probable vascular dementia or Alzheimer's disease with cerebrovascu- 
lar disease. Eur J Neurol 2003;10:63340.

16. Thorvaldsen P. Stroke incidence, case fatality, and mortality in the WHO MONICA project. Stroke 1995;26:3617.

17. Román GC. Vascular dementia revisited: diagnosis, pathogenesis, treatment, and prevention. Med Clin N Am 2002;86:477-99.

18. World Health Organization. The ICD10 classification of mental and behavioural disorders: clinical descriptions and diagnostic guidelines. Geneva: World Health Organization; 1992.

19. Hatano S. Experience from a multicentre stroke register: a preliminary report. Bull World Health Organ 1976;54:54153.

20. Goldstein LB, Bertels C, Davis JN. Interrater reliability of the nih stroke scale. Archiv Neurol 1989;46:660-2.

21. Health M. Manual of elderly screening and evaluation Nonthaburi: the War Veterans Press of Thailand; 2013.
22. Limpawattana P. Can Rowland Universal Dementia Assessment Scale (RUDAS) Replace Mini-Mental State Examination (MMSE) for Dementia Screening in a Thai Geriatric Outpatient Setting? Am J Alzheim Dis Other Dement 2012;27:254-9.

23. Sunderland T. Diagnostic issues in dementia: advancing the research agenda for DSM-V. American Psychiatric Pub; 2008.

24. Chobanian AV. The seventh report of the joint national committee on prevention, detection, evaluation, and treatment of high blood pressure: the JNC 7 report. JAMA 2003;289:2560-71.

25. Alberti KGMM, Zimmet PF. Definition, diagnosis and classification of diabetes mellitus and its complications. Part 1: diagnosis and classification of diabetes mellitus. Provisional report of a WHO consultation. Diabet Med 1998;15:539-53.

26. Williams L. Third report of the National Cholesterol Education Program
(NCEP) expert panel on detection, evaluation, and treatment of high blood cholesterol in adults (Adult Treatment Panel III) final report. Circulation 2002;106:3143-4.

27. Jacquin A. Post-stroke cognitive impairment: high prevalence and determining factors in a cohort of mild stroke. J Alzheimers Dis 2014;40:102938.

28. Mellon L. Cognitive impairment six months after ischaemic stroke: a profile from the ASPIRE-S study. BMC Neurol 2015;15:31.

29. Katon WJ. Comorbid depression is associated with an increased risk of dementia diagnosis in patients with diabetes: a prospective cohort study. J Gener Intern Med 2010;25:423-9.

30. Surawan J. Risk factors associated with post-stroke dementia: a systematic review and meta-analysis. Neurol Int 2017;9:7216. 